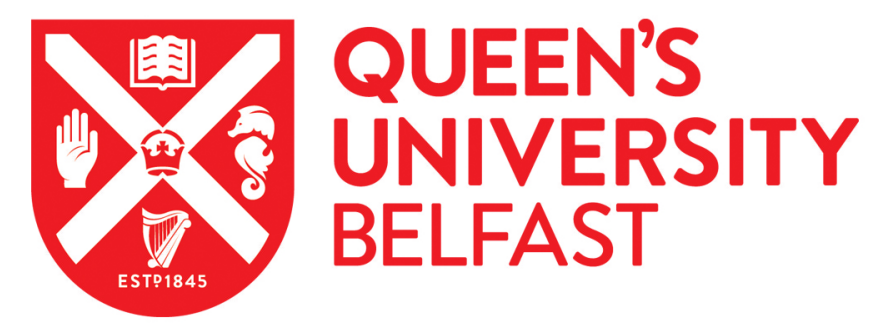

\title{
The BER Analysis of MRC-aided Greedy Detection for OFDM-IM in Presence of Uncertain CSI
}

Luong, T. V., \& Ko, Y. (2018). The BER Analysis of MRC-aided Greedy Detection for OFDM-IM in Presence of Uncertain CSI. IEEE Wireless Communications Letters. https://doi.org/10.1109/LWC.2018.2797066

\author{
Published in: \\ IEEE Wireless Communications Letters
}

Document Version:

Peer reviewed version

Queen's University Belfast - Research Portal:

Link to publication record in Queen's University Belfast Research Portal

Publisher rights

(c) 2018 IEEE.

This work is made available online in accordance with the publisher's policies. Please refer to any applicable terms of use of the publisher.

\section{General rights}

Copyright for the publications made accessible via the Queen's University Belfast Research Portal is retained by the author(s) and / or other copyright owners and it is a condition of accessing these publications that users recognise and abide by the legal requirements associated with these rights.

Take down policy

The Research Portal is Queen's institutional repository that provides access to Queen's research output. Every effort has been made to ensure that content in the Research Portal does not infringe any person's rights, or applicable UK laws. If you discover content in the Research Portal that you believe breaches copyright or violates any law, please contact openaccess@qub.ac.uk. 


\title{
The BER Analysis of MRC-aided Greedy Detection for OFDM-IM in Presence of Uncertain CSI
}

\author{
Thien Van Luong, Student Member, IEEE, and Youngwook Ko, Senior Member, IEEE
}

\begin{abstract}
This letter investigates the bit error rate (BER) performance of orthogonal frequency division multiplexing index modulation, employing the maximal ratio combining based lowcomplexity greedy detector (MRC-GD) and the PSK modulation. For performance analysis, we derive tight expressions for both index error probability (IEP) and BER, taking into account channel state information (CSI) uncertainty. This allows to provide an insight into various impacts of CSI uncertainty on the diversity gain and error floor of the IEP and the BER, respectively. We clearly show that under imperfect CSI, the MRC-aided GD can perform as like the MRC-maximum likelihood detector, at lower complexity. Finally, simulation results are presented to verify the accuracy of derived expressions and the theoretical analysis.
\end{abstract}

Index Terms-OFDM-IM, index modulation, maximal ratio combining (MRC), bit error rate, imperfect CSI.

\section{INTRODUCTION}

Orthogonal frequency division multiplexing with index modulation (OFDM-IM) [1] is an attractive multicarrier scheme with higher energy efficiency and reliability over the conventional OFDM. In particular, OFDM-IM transmits data bits via not only the classical $M$-ary symbols but also the indices of sub-carriers activated [2]. Thus, OFDM-IM can provides an appealing trade-off between the spectral efficiency (SE) and the error performance just by adjusting the number of active sub-carriers.

A variety of IM concepts have been studied aiming to enhance the SE or reliability of systems, which can be found in the survey [3]. Notice that most of existing works investigate the bit error rate (BER) of OFDM-IM with the maximum likelihood (ML) detection [1], [2]. To reduce the complexity of the ML, the log-likelihood ratio (LLR) [1] and modified ML [4] detectors are studied. Recently, a low-complexity greedy detector (GD) based on energy detection is proposed in [5]. The symbol error probability (SEP) and BER of this detector in presence of channel estimation information (CSI) uncertainty are presented in [6], [7], respectively. In [8], OFDM-IM with the hybrid maximal ratio combining and greedy detection (MRC-GD) is proposed, where the SEP is analyzed, but only for perfect CSI. Since [6], [7] clearly show that the GD is less sensitive to CSI uncertainty than the ML, it is worthy to investigate how the low complexity MRC-GD detector helps to decrease the sensitivity to CSI uncertainty.

In this letter, we provide a novel approach to derive the index error probability (IEP) of OFDM-IM with MRC-GD

The authors are with School of Electronics, Electrical Eng. and Computer Science, Queen's University of Belfast, United Kingdom (Email: \{tluong01, y.ko\}@qub.ac.uk). This work was supported by the Engineering and Physical Sciences Research Council [grant number EP/N509541/1] and the PSK modulation, in the presence of CSI uncertainty, which is then used to obtain the tight expression for the BER. Analyzing derived expressions, we clearly show that due to CSI uncertainty, the diversity order of the IEP is limited to one, even with the MRC-aided GD, which is unlike previous works [6], [7] where the IEP of the GD is not affected by CSI uncertainty. Interestingly, we also find that the BER suffers from an error floor, the level of which can decrease exponentially with the number of antennas. Hence, for OFDMIM applications especially under uncertain CSI, the MRC-GD is desired, obtaining the BER close to the MRC-ML detector. ${ }^{1}$

\section{System Model}

\section{A. OFDM-IM}

Consider an OFDM-IM system where the transmitter has a single antenna, while the receiver has $L$ antennas. There are a total of $N_{c}$ sub-carriers that are partitioned into $G$ clusters of $N$ sub-carriers. Due to the independent operation of each cluster, we consider only one cluster hereinafter for simplicity.

In every transmission, $K$ out of $N$ sub-carriers are activated to carry data bits via not only complex $M$-ary symbols but also indices of the active sub-carriers. Particularly, $p$ incoming bits are divided into two groups of $p_{1}$ and $p_{2}$ bits. Based on the look-up table or combinatorial method [1], the first $p_{1}$ index bits are mapped to a set of $K$ active sub-carrier indices which is denoted by $\theta=\left\{\alpha_{1}, \ldots, \alpha_{K}\right\}$, where $\alpha_{k} \in\{1, \ldots, N\}$ for $k=1, \ldots, K$. Notice that $\theta$ can be considered as an index symbol. The $p_{2}$ data symbol bits are mapped to $K$ non-zero $M$-ary symbols. As a result, we obtain $p_{1}=\left\lfloor\log _{2} C(N, K)\right\rfloor$ and $p_{2}=K \log _{2} M$. Based on the $K$ symbols and the index set $\theta$ defined, the transmitted signal is generated as $\mathbf{x}=[x(1), \ldots, x(N)]^{T}$, where $x(\alpha)=0$ for $\alpha \notin \theta$ and $x(\alpha) \in \mathcal{S}$ for $\alpha \in \theta$, where $\mathcal{S}$ denotes the PSK constellation.

In the frequency domain, the received signals from $L$ antennas for each sub-carrier $\alpha$ is given by

$$
\mathbf{y}_{\alpha}=\mathbf{h}_{\alpha} x(\alpha)+\mathbf{n}_{\alpha}, \quad \alpha=1, \ldots, N
$$

where $\mathbf{h}_{\alpha}=\left[h_{1}(\alpha), \ldots, h_{L}(\alpha)\right]^{T}$ denotes the Rayleigh fading channel vector between the transmitter and $L$ receiver antennas with $h_{l}(\alpha) \sim \mathcal{C N}(0,1)$, and $\mathbf{n}_{\alpha}$ is the noise vector with its elements $n_{l}(\alpha) \sim \mathcal{C N}\left(0, N_{0}\right)$. Denote by $\varphi E_{s}$ the average

\footnotetext{
${ }^{1}$ Column vectors and matrices are denoted by lower and upper case boldface letters, respectively. $(.)^{T}$ and $\lfloor$.$\rfloor denote transposition and the floor function,$ respectively. $\mathcal{C N}\left(0, \sigma^{2}\right)$ is the complex Gaussian distribution with zero mean and variance $\sigma^{2}$, while $\mathcal{N}\left(0, \sigma^{2}\right)$ is the real-valued Gaussian distribution. $\mathbb{E}\{$.$\} and C(.,$.$) are expectation and the binomial coefficient, respectively.$ $\Gamma(\kappa, \beta)$ is the Gamma distribution with the scale parameter $\beta$ and shape parameter $\kappa . I_{0}($.$) is the zero order modified Bessel function of first kind.$
} 
power per non-zero $M$-ary symbol, where $\varphi=N / K$ is the power allocation ratio. Hence, the average signal-to-noise ratio (SNR) per active sub-carrier is $\bar{\gamma}=\varphi E_{s} / N_{0}$.

\section{B. Post-MRC Greedy Detector under CSI Uncertainty}

In practical systems, the true channel $\mathbf{h}_{\alpha}$ in (1) is uncertainly estimated into $\hat{\mathbf{h}}_{\alpha}=\left[\hat{h}_{1}(\alpha), \ldots, \hat{h}_{L}(\alpha)\right]^{T}$ which satisfies [6]

$$
\mathbf{h}_{\alpha}=\hat{\mathbf{h}}_{\alpha}+\mathbf{e}_{\alpha},
$$

where $\mathbf{e}_{\alpha}$ denotes the channel estimation error with its elements $e_{l}(\alpha) \sim \mathcal{C N}\left(0, \epsilon^{2}\right)$ being independent of $\hat{h}_{l}(\alpha)$, and $\hat{h}_{l}(\alpha) \sim \mathcal{C N}\left(0,1-\epsilon^{2}\right)$, where $\epsilon^{2} \in[0,1)$ represents the error variance. Using $\hat{\mathbf{h}}_{\alpha}$ and the MRC as $y(\alpha)=\hat{\mathbf{h}}_{\alpha}^{H} \mathbf{y}_{\alpha}$, the combined signal at sub-carrier $\alpha$ is

$$
y(\alpha)=h(\alpha) x(\alpha)+n(\alpha),
$$

where $h(\alpha)=\hat{\mathbf{h}}_{\alpha}^{H} \mathbf{h}_{\alpha}$ and $n(\alpha)=\hat{\mathbf{h}}_{\alpha}^{H} \mathbf{n}_{\alpha}$.

The low complexity greedy detector (GD) uses the output of the MRC, i.e., $y(\alpha)$ to detect signals via two steps. Firstly, the GD estimates the active indices $\hat{\alpha}$ that are corresponding to $K$ sub-carriers having largest normalized MRC-output energies, i.e., $|\bar{y}(\alpha)|^{2}$, where $\bar{y}(\alpha)=y(\alpha) / T_{\alpha}$ with $T_{\alpha}=\hat{\mathbf{h}}_{\alpha}^{H} \hat{\mathbf{h}}_{\alpha}$. Secondly, $K$ non-zero symbols are decoded using the ML criterion to active sub-carrier $\hat{\alpha}$ as

$$
x(\hat{\alpha})=\arg \min _{x(\hat{\alpha}) \in \mathcal{S}}\left|y(\hat{\alpha})-T_{\alpha} x(\hat{\alpha})\right|^{2} .
$$

It should be noted that the normalization of $y(\alpha)$ is necessary to suppress severe effects of $\hat{\mathbf{h}}_{\alpha}^{H}$ on the index detection process, which is not clearly presented in [8] (Section II.B). This significantly affects the accuracy of the IEP derivation as shown in the next section.

Note that in [6], the GD is proved to be less affected by CSI uncertainty than the ML. However, for OFDM-IM with single antenna, the ML still considerably outperforms the GD under certain CSI conditions when $M$ is small $(M=2,4)$. This might be no longer true as the number of antennas $L$ increases. These motivate us to analyze the BER of OFDM-IM when using the MRC-GD in the presence of CSI uncertainty.

\section{BER ANALYSIS UNDER CSI UNCERTAINTY}

Follow the approach in [7], we first derive the IEP of OFDM-IM with MRC-GD, considering CSI uncertainty. After the evaluation of IEP, the BER expression will be provided.

\section{A. Index Error Probability}

An index symbol error occurs when the index set $\theta$ is incorrectly detected. Hence, the instantaneous IEP (iIEP) can be given by [6]

$$
P_{I} \leq \frac{K}{N} \sum_{\alpha=1}^{N} \sum_{\tilde{\alpha} \neq \alpha=1}^{N-K} P(\alpha \rightarrow \tilde{\alpha})
$$

where $P(\alpha \rightarrow \tilde{\alpha})$ is the pairwise error probability (PEP) that active sub-carrier $\alpha$ is incorrectly estimated as inactive one $\tilde{\alpha} \neq \alpha$. Based on the first step of the GD, the PEP is given by

$$
P(\alpha \rightarrow \tilde{\alpha})=P\left\{|\bar{y}(\tilde{\alpha})|^{2}>|\bar{y}(\alpha)|^{2}\right\}
$$

Note that while [6]-[8] simply reuse the iIEP expression from [5], this is impossible for the MRC-GD with uncertain CSI. Thus, we develop a novel analytical approach to derive the iIEP and its average of MRC-GD with uncertain CSI, which are more accurate than that in [8]. In particular, the iIEP is obtained using (5)-(6) in the following lemma.

Lemma 1: The iIEP expression of OFDM-IM with MRC-GD under CSI uncertainty is given by

$$
P_{I} \leq \frac{K}{N} \sum_{\alpha=1}^{N} \sum_{\tilde{\alpha} \neq \alpha=1}^{N-K} \frac{T_{\alpha}}{\delta T_{\tilde{\alpha}}+T_{\alpha}} e^{-\frac{\bar{\gamma} T_{\tilde{\alpha}} T_{\alpha}}{\delta T_{\tilde{\alpha}}+T_{\alpha}}},
$$

where $\delta=1+\bar{\gamma} \epsilon^{2}$ and $\tilde{\alpha} \neq \alpha$.

Proof: See Appendix A.

Notice that the iIEP expression in (7) is totally different from that in [8, Eq. (10)], in which normalized MRC-output energies are not taken into account as our derivation. In fact, this makes [8] yield a less accurate IEP (under perfect CSI), even in high SNR regions. By contrast, our proposed approach provides the tighter IEP expression, even at low SNRs, under both perfect and imperfect CSI, as in the following theorem.

Theorem 1: Consider OFDM-IM with MRC-GD detection. The average IEP under CSI uncertainty is given by

$$
\bar{P}_{I} \leq \frac{\delta^{L} \Upsilon(2 L-1) !}{[(L-1) !]^{2}} \int_{0}^{1} \frac{x^{L}(1-x)^{L-1} d x}{\left(1+\bar{\gamma} x-\beta \bar{\gamma} x^{2}\right)^{2 L}}
$$

where $\Upsilon=K(N-K), \beta=1-\epsilon^{2}$ and $\delta=1+\bar{\gamma} \epsilon^{2}$.

Proof: See Appendix B.

As seen from (8), the IEP of MRC-GD depends on $\epsilon^{2}$, while that of the GD with single antenna [6] is not affected by $\epsilon^{2}$. Moreover, the derivation of the average IEP in Theorem 1 for the MRC-GD under uncertain CSI is novel, and more challenging than that in [8] with perfect CSI, which is straightforward from [5]. Finally, the following corollary provides an insight into the impact of $\epsilon^{2}$ on $\bar{P}_{I}$.

Corollary 1: For given $\epsilon^{2}>0$ and at high SNRs, we obtain $\bar{P}_{I} \approx(\kappa \bar{\gamma})^{-1}$ where $\kappa$ is constant.

Proof: At large $\bar{\gamma}$ and for given $\epsilon^{2}>0$, notice from (8) that $x \in(0,1)$, we can approximate $\delta^{L} \approx\left(\epsilon^{2} \bar{\gamma}\right)^{L}$ and $1+\bar{\gamma} x-\beta \bar{\gamma} x^{2} \approx 1+\bar{\gamma} x$. Thus, we attain $\bar{P}_{I} \approx$ $A B$, where $A=\Upsilon \epsilon^{2 L}(2 L-1) ! /[(L-1) !]^{2}$, and $B=$ $\bar{\gamma}^{L} \int_{0}^{1} \frac{x^{L}(1-x)^{L-1} d x}{(1+\bar{\gamma} x)^{2 L}}$ which relies on $\bar{\gamma}$. Let $u=1+\bar{\gamma} x$, then $B$ can be approximated, at high SNRs, by $B \approx C \bar{\gamma}^{-1}$, where $C=\int_{1}^{1+\bar{\gamma}} \frac{(u-1)^{L}(1-u / \bar{\gamma})^{L-1} d u}{u^{2 L}}$ which is constant when $\bar{\gamma}$ tends to infinity. As a result, we obtain $\bar{P}_{I} \approx(\kappa \bar{\gamma})^{-1}$ where $\kappa^{-1}=A C$.

Remark 1: Corollary 1 shows that the MRC-GD achieves unit diversity order in terms of the average IEP for any $\epsilon^{2}>0$.

\section{B. Bit Error Rate}

Following the approach in [7] and utilizing $\bar{P}_{I}$, the BER of the MRC-GD (denoted by $P_{b}$ ) can be approximated by

$$
P_{b} \approx \frac{\left(\eta p_{1}+m\right) \bar{P}_{I} / 2+K \bar{P}_{M}}{p_{1}+p_{2}},
$$

where $\bar{P}_{M}$ is the average SEP of classical $M$-ary symbols (4) when active indices are correctly detected, and $m=\log _{2} M$, 
$\eta=1,2$ for $N>2$ and $N=2$, respectively. If the $M$-PSK modulation is used, $\bar{P}_{M}$ is provided by the following lemma.

Lemma 2: Consider OFDM-IM with MRC-GD detection. The average SEP of the classical $M$-ary symbols is given by

$$
\bar{P}_{M} \approx \frac{\xi}{12}\left[\frac{1}{(1+\beta \bar{\gamma} \rho / \delta)^{L}}+\frac{3}{(1+4 \beta \bar{\gamma} \rho / 3 \delta)^{L}}\right],
$$

where $\xi=1,2$ for $M=2$ and $M>2$, respectively, and $\rho=\sin ^{2}(\pi / M)$.

Proof: See Appendix C.

Finally, inserting (8) and (10) into (9), the BER of MRC-GD can be expressed as

$$
\begin{aligned}
P_{b} & \approx \frac{\left(\eta p_{1}+m\right) \delta^{L} \Upsilon(2 L-1) !}{2 p[(L-1) !]^{2}} \int_{0}^{1} \frac{x^{L}(1-x)^{L-1} d x}{\left(1+\bar{\gamma} x-\beta \bar{\gamma} x^{2}\right)^{2 L}} \\
& +\frac{K \xi}{12 p}\left[\frac{1}{(1+\beta \bar{\gamma} \rho / \delta)^{L}}+\frac{3}{(1+4 \beta \bar{\gamma} \rho / 3 \delta)^{L}}\right] .
\end{aligned}
$$

Remark 2: As seen from (11), for given $\epsilon^{2}>0$, $\lim _{\bar{\gamma} \rightarrow \infty} \beta \bar{\gamma} \rho / \delta=\beta \rho / \epsilon^{2}>0$ and $\lim _{\bar{\gamma} \rightarrow \infty} \bar{P}_{I}=0$ (see Corollary 1). Thus, there exists an error floor on the BER which is defined only by $M$-ary symbol detection errors as

$$
\lim _{\bar{\gamma} \rightarrow \infty} P_{b}=\Upsilon\left[(1+\mu)^{-L}+3(1+4 \mu / 3)^{-L}\right],
$$

where $\Upsilon=K \xi / 12 p$ and $\mu=\beta \rho / \epsilon^{2}$. It is worth noting that the error floor in (12) decreases exponentially with $L$.

Remark 3: It is shown from (9) that $\bar{P}_{M}$ is the same for both the MRC-ML and the MRC-GD. Thus, when $K$ increases to $N$, the BERs of both detectors tend to that of the classical OFDM with MRC, relying much more on the $M$-ary symbol errors and less on the index errors. The intuition behind this is that for large $K, p_{1}$ is relatively small and $\bar{P}_{I}$ in (8) gets negligible.

\section{NUMERICAL AND SIMULATION RESUlTS}

We present simulation and theoretical results for the IEP and BER of OFDM-IM with MRC-GD under both perfect and imperfect CSI. Particularly, we consider OFDM-IM with $N \in\{2,4\}, K \in\{1,2\}, M \in\{2,4\}$ and $L \in\{1,2,4,6,8\}$. The performance of the MRC-ML is used for comparison.

Fig. 1 depicts the average IEP and BER of OFDM-IM with MRC-GD/MRC-ML under both perfect $\left(\epsilon^{2}=0\right)$ and imperfect CSI $\left(\epsilon^{2}=0.1\right)$. As seen from Fig. 1, the theoretical bounds are accurate in a range of SNRs. In addition, for given $L$, the CSI uncertainty significantly degrades the performance of MRC-GD, especially at high SNRs. For example, in Fig. 1.a, the average IEPs at high SNRs are shown to have the diversity order of one, for any $L$ and $\epsilon^{2}=0.1$, which validates Corollary 1 and Remark 1. In Fig. 1.b, we can see the error floors on the BER caused by $\epsilon^{2}=0.1$. Moreover, when $L$ increases, the error floor decreases substantially. These confirm Remark 2. Compared to the MRC-ML, it is shown via Fig. 1.b that under the CSI uncertainty, the BER performance gap between two detectors is marginal, especially at high SNRs. Meanwhile, under perfect CSI, the BER of the MRC-GD tends to that of the MRC-ML for increasing $L$.
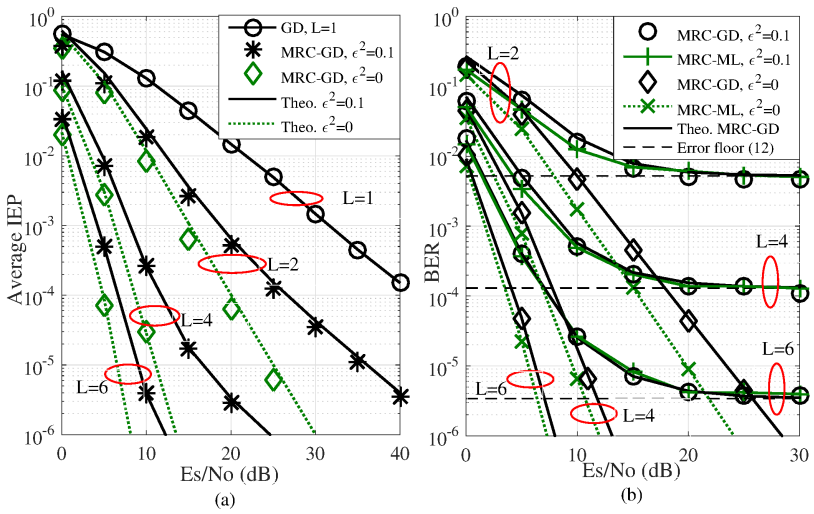

Fig. 1. Average IEP (a) and BER (b) of MRC-GD under perfect and imperfect CSI, when $(N, K, M)=(4,2,4), L \in\{1,2,4,6\}$ and $\epsilon^{2} \in\{0,0.1\}$.
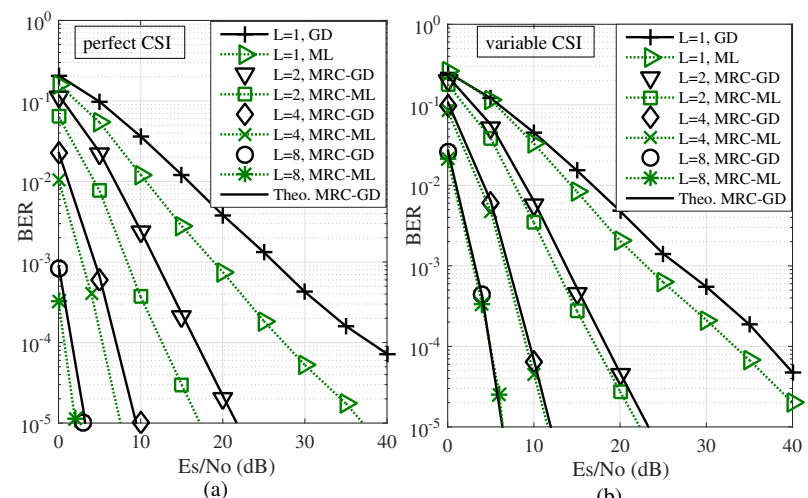

Fig. 2. BER comparison between MRC-GD and MRC-ML under (a) perfect CSI and (b) variable CSI, when $(N, K, M)=(2,1,2)$ and $L \in\{1,2,4,8\}$.

In Fig. 2, we compare the BER between the two detectors under perfect and variable CSI, where $\epsilon^{2}$ varies as a decreasing function of the SNR, i.e., $\epsilon^{2}=1 /\left(1+E_{s} / N_{0}\right)$ [7]. In Fig. 2.a, the MRC-ML still significantly outperforms the MRC-GD for small $L(L=1,2)$. However, this is no longer true in Fig. 2.b with variable CSI, where the gaps between the two detectors are negligible $(<0.5 \mathrm{~dB})$ for any $L \geq 2$.

\section{CONCLUSIONS}

We derived the tight expression for the BER of OFDM-IM with MRC-GD in the presence of CSI uncertainty. Particularly, impacts of CSI uncertainty on both the IEP and the BER were analyzed. We found out that for given $\epsilon^{2}>0$, the index bits detection suffers from the limited diversity order to be one for any number of antennas $L$, while the BER has the error floor decreasing exponentially with $L$. Interestingly, under imperfect CSI, the performance gap between the MRCGD and the MRC-ML is less than $0.5 \mathrm{~dB}$ as $L \geq 2$, even for small $M$, i.e., $M=2,4$. Hence, for OFDM-IM applications, the MRC-GD is preferable to the MRC-ML, especially under uncertain CSI and at larger $L$. 


\section{APPENDIX}

\section{A. Proof of Lemma 1}

From (6), let $Y_{\alpha}=|\bar{y}(\alpha)|^{2}$ and $Z_{\tilde{\alpha}}=|\bar{y}(\tilde{\alpha})|^{2}$. Due to $x(\tilde{\alpha})=0$, using (3) we have $\bar{y}(\tilde{\alpha})=n(\tilde{\alpha}) / T_{\tilde{\alpha}} \sim$ $\mathcal{C N}\left(0, N_{0} / T_{\tilde{\alpha}}\right)$ with $T_{\tilde{\alpha}}=\hat{\mathbf{h}}_{\tilde{\alpha}}^{H} \hat{\mathbf{h}}_{\tilde{\alpha}}$. This results in $Z_{\tilde{\alpha}} \sim$ $\Gamma\left(1,1 / \widehat{T}_{\tilde{\alpha}}\right)$ with $\widehat{T}_{\tilde{\alpha}}=T_{\tilde{\alpha}} / N_{0}$. Thus, the probability density function (PDF) of $Z_{\tilde{\alpha}}$ is

$$
F_{Z_{\tilde{\alpha}}}(x)=1-e^{-\hat{T}_{\tilde{\alpha}} x} .
$$

Regarding the PDF of $Y_{\alpha}$, notice from (3) and (2) that $h(\alpha)=\hat{\mathbf{h}}_{\alpha}^{H}\left(\hat{\mathbf{h}}_{\alpha}+\mathbf{e}_{\alpha}\right)=T_{\alpha}+\hat{\mathbf{h}}_{\alpha}^{H} \mathbf{e}_{\alpha}$, we can represent $\bar{y}(\alpha)$ as $\bar{y}(\alpha)=x(\alpha)+\bar{n}(\alpha)$, where $\bar{n}(\alpha)=\tilde{n}(\alpha) / T_{\alpha} \sim$ $\mathcal{C N}\left(0, \widetilde{N}_{0} / T_{\alpha}\right)$ with $\tilde{n}(\alpha)=\hat{\mathbf{h}}_{\alpha}^{H}\left[\mathbf{e}_{\alpha} x(\alpha)+\mathbf{n}_{\alpha}\right]$ and $\tilde{N}_{0}=$ $\varphi E_{s} \epsilon^{2}+N_{0}$. Assume that $x(\alpha)=\sqrt{\varphi E_{s}} e^{j \phi}$. Hence, $\bar{y}(\alpha)$ can be rewritten by $\bar{y}(\alpha)=e^{j \phi}\left[\sqrt{\varphi E_{s}}+e^{-j \phi} \bar{n}(\alpha)\right]$. Since $e^{-j \phi} \bar{n}(\alpha) \sim \mathcal{C N}\left(0,1 / \widehat{T}_{\alpha}\right)$ where $\widehat{T}_{\alpha}=T_{\alpha} / \widetilde{N}_{0}$, we can assume $e^{-j \phi} \bar{n}(\alpha)=a+j b$ where $a, b \sim \mathcal{N}\left(0,1 / 2 \widehat{T}_{\alpha}\right)$ to obtain $Y_{\alpha}=c^{2}+b^{2}$, where $c=\sqrt{\varphi E_{s}}+a \sim$ $\mathcal{N}\left(\sqrt{\varphi E_{s}}, 1 / 2 \widehat{T}_{\alpha}\right)$. Notice that $U_{\alpha}=2 \widehat{T}_{\alpha} Y_{\alpha}$ is the noncentral chi-squared distribution with degree of freedoms of two and the non-centrality parameter of $\lambda=2 \widehat{T}_{\alpha} \varphi E_{s}$, i.e., $U_{\alpha} \sim \mathcal{X}_{2}^{2}(\lambda)$ [9]. As a result, the PDF of $Y_{\alpha}$ can be obtained using $f_{Y_{\alpha}}(x)=2 \widehat{T}_{\alpha} f_{U_{\alpha}}\left(2 \widehat{T}_{\alpha} x\right)$ as

$$
f_{Y_{\alpha}}(x)=\widehat{T}_{\alpha} e^{-\widehat{T}_{\alpha}\left(\varphi E_{s}+x\right)} I_{0}\left(2 \widehat{T}_{\alpha} \sqrt{\varphi E_{s} x}\right) .
$$

From (6), the PEP is calculated using (13) and (14) as [5]

$$
\begin{aligned}
P(\alpha \rightarrow \tilde{\alpha}) & =P\left\{Z_{\tilde{\alpha}}>Y_{\alpha}\right\}=\int_{0}^{\infty} \int_{y}^{\infty} f_{Y_{\alpha}}(y) f_{Z_{\tilde{\alpha}}}(z) d y d z \\
& =\widehat{T}_{\alpha} \int_{0}^{\infty} e^{-\left(\hat{T}_{\tilde{\alpha}}+\widehat{T}_{\alpha}\right) y-\varphi E_{s} \widehat{T}_{\alpha}} I_{0}\left(2 \widehat{T}_{\alpha} \sqrt{\varphi E_{s} y}\right) d y
\end{aligned}
$$

Finally, the PEP in (15) can be attained with the aid of [10, 6.614-3], which leads to the instantaneous IEP (5) obtained after some manipulations as (7).

\section{B. Proof of Theorem 1}

Let $X_{1}=T_{\tilde{\alpha}}$ and $X_{2}=T_{\alpha} / \delta T_{\tilde{\alpha}}+T_{\alpha}$. The average IEP can be obtained by averaging (7) over $X_{1}$ and $X_{2}$ as

$$
\bar{P}_{I} \leq K(N-K) \mathbb{E}_{X_{1}, X_{2}}\left\{X_{2} e^{-\bar{\gamma} X_{1} X_{2}}\right\} .
$$

Let $r=\mathbb{E}_{X_{1}, X_{2}}\left\{X_{2} e^{-\bar{\gamma} X_{1} X_{2}}\right\}$. Notice that $X_{2} \in(0,1)$, thus $r$ can be expressed by

$$
r=\int_{0}^{\infty} \int_{0}^{1} f_{X_{1}, X_{2}}\left(x_{1}, x_{2}\right) x_{2} e^{-\bar{\gamma} x_{1} x_{2}} d x_{1} d x_{2},
$$

which prompts us to derive $f_{X_{1}, X_{2}}\left(x_{1}, x_{2}\right)$ as follows.

We represent $T_{\alpha}$ and $T_{\tilde{\alpha}}$ with respect to $X_{1}$ and $X_{2}$ as $T_{\tilde{\alpha}}=$ $X_{1}$ and $T_{\alpha}=\delta X_{1} X_{2} /\left(1-X_{2}\right)$. This results in $\partial T_{\tilde{\alpha}} / \partial X_{1}=$ 1, $\partial T_{\tilde{\alpha}} / \partial X_{2}=0$ and $\partial T_{\alpha} / \partial X_{2}=\delta X_{1} /\left(1-X_{2}\right)^{2}$. As a result, the Jacobian determinant is given by $J\left(x_{1}, x_{2}\right)=$ $\delta x_{1} /\left(1-x_{2}\right)^{2}$ [9]. Moreover, due to the system model, we have $f_{T_{\tilde{\alpha}}}(x)=f_{T_{\alpha}}(x)=x^{L-1} e^{-\frac{x}{\beta}} /(L-1) ! \beta^{L}$. Here, using the Jacobian transformation, we obtain

$$
\begin{aligned}
f_{X_{1}, X_{2}}\left(x_{1}, x_{2}\right) & =f_{T_{\tilde{\alpha}}}\left(x_{1}\right) f_{T_{\alpha}}\left(\frac{\delta x_{1} x_{2}}{1-x_{2}}\right) J\left(x_{1}, x_{2}\right) \\
& =\frac{\delta^{L} \beta^{-2 L}}{[(L-1) !]^{2}} \times \frac{x_{1}^{2 L-1} x_{2}^{L-1} e^{-\frac{x_{1}}{\beta}\left(1+\frac{\delta x_{2}}{1-x_{2}}\right)}}{\left(1-x_{2}\right)^{L+1}} .
\end{aligned}
$$

Plugging (18) into (17) leads to

$$
r=\Psi \int_{0}^{1} \frac{x_{2}^{L} \int_{0}^{\infty} x_{1}^{2 L-1} e^{-\frac{x_{1}}{\beta}\left(1+\frac{\delta x_{2}}{1-x_{2}}+\beta \bar{\gamma} x_{2}\right)} d x_{1}}{\left(1-x_{2}\right)^{L+1}} d x_{2}
$$

where $\Psi=\delta^{L} / \beta^{2 L}[(L-1) !]^{2}$. With the aid of [10, 3.381-3], i.e., $\int_{0}^{\infty} x^{n} e^{-a x} d x=n ! / a^{n+1}$ and after simple manipulations, $r$ in (19) is given by

$$
r=\frac{(2 L-1) ! \delta^{L}}{[(L-1) !]^{2}} \int_{0}^{1} \frac{x_{2}^{L}\left(1-x_{2}\right)^{L-1} d x_{2}}{\left(1+\bar{\gamma} x_{2}-\beta \bar{\gamma} x_{2}^{2}\right)^{2 L}}
$$

Finally, $\bar{P}_{I}$ is obtained by substituting (20) to (16).

\section{Proof of Lemma 2}

It is seen from (4) that under imperfect CSI, $x(\alpha)$ is detected with an instantaneous SNR of $\gamma_{\alpha}=$ $\varphi E_{s} T_{\alpha} /\left(\varphi E_{s} \epsilon^{2}+N_{0}\right)=\bar{\gamma} T_{\alpha} / \delta$. Thus, the instantaneous SEP of the $M$-ary PSK symbols is given by [7]

$$
P_{M}(\alpha) \approx \frac{\xi}{12}\left(e^{-\bar{\gamma} T_{\alpha} \rho / \delta}+3 e^{-4 \bar{\gamma} T_{\alpha} \rho / 3 \delta}\right),
$$

where $\rho=\sin ^{2}(\pi / M)$, and $\xi=1,2$ for $M=2$ and $M>2$.

Notice that the moment generating function (MGF) of $T_{\alpha}$ given by $\mathcal{M}_{T_{\alpha}}(t)=(1-\beta t)^{-L}$. Finally, using MGF approach to (21), we easily obtain $\bar{P}_{M}$ as in (10).

\section{REFERENCES}

[1] E. Basar, U. Aygolu, E. Panayirci, and H. V. Poor, "Orthogonal frequency division multiplexing with index modulation," IEEE Trans. Signal Process., vol. 61, no. 22, pp. 5536-5549, Nov 2013.

[2] Y. Ko, "A tight upper bound on bit error rate of joint OFDM and multicarrier index keying," IEEE Commun. Lett., vol. 18, no. 10, pp. 17631766, Oct 2014.

[3] E. Basar, M. Wen, R. Mesleh, M. D. Renzo, Y. Xiao, and H. Haas, "Index modulation techniques for next-generation wireless networks," IEEE Access, vol. 5, pp. 16693-16746, 2017.

[4] M. Wen, B. Ye, E. Basar, Q. Li, and F. Ji, "Enhanced orthogonal frequency division multiplexing with index modulation," IEEE Trans. Wireless Commun., vol. PP, no. 99, pp. 1-1, 2017.

[5] J. Crawford and Y. Ko, "Low complexity greedy detection method with generalized multicarrier index keying OFDM," in Proc. IEEE Pers., Indoor., Mobile Radio Commun., Aug 2015, pp. 688-693.

[6] T. V. Luong and Y. Ko, "Impact of CSI uncertainty on MCIK-OFDM: tight, closed-form symbol error probability analysis," IEEE Trans. Veh. Technol., vol. PP, no. 99, pp. 1-1, 2017.

[7] - "A tight bound on BER of MCIK-OFDM with greedy detection and imperfect CSI," IEEE Commun. Lett., vol. PP, no. 99, pp. 1-1, Aug 2017.

[8] J. Crawford, E. Chatziantoniou, and Y. Ko, "On the SEP analysis of OFDM index modulation with hybrid low complexity greedy detection and diversity reception," IEEE Trans. Veh. Technol., vol. 66, no. 9, pp. 8103-8118, Sep. 2017.

[9] N. Johnson, S. Kotz, and N. Balakrishnan, Continuous univariate distributions. Wiley \& Sons, 1995, no. Volume 2.

[10] I. Gradshteyn and I. Ryzhik, Table of Integrals, Series, and Products, A. Jeffrey and D. Zwillinger, Eds. Academic Press, 2007. 